\title{
MULTISCALE MODELLING OF FLOW DUE TO THE PERISTALTIC WAVE IN DEFORMING PORO-PIEZOELECTRIC MEDIUM
}

\author{
EDUARD ROHAN* AND VLADIMÍR LUKEŠ* \\ ${ }^{*}$ Department of Mechanics \& NTIS New Technologies for Information Society, Faculty of \\ Applied Sciences, University of West Bohemia in Pilsen, \\ Univerzitní 22, 30100 Plzeň, Czech Republic \\ rohan@kme.zcu.cz
}

Key words: Multiscale Modelling, Porous Media, Homogenization, Piezoelectric Memtamaterial, Peristaltic Flow

\begin{abstract}
The paper reports on the homogenization based modelling of fluid saturated poroelastic materials containing locally controlable piezoelectric (PZ) actuators. This option provides metamaterial properties which enable to convert the electric power into the fluid transport due to the peristaltic deformation wave induced by the propagating voltage wave. A quasi-linear PZ-poroelastic material model is proposed to respect dependence of the effective medium parameters on the deformation at the microstructure (pore) level. Due to the sensitivity analysis of the homogenized coefficient, the two-scale modelling avoids any need to update the local microconfigurations. Numerical studies has been performed as the proof of the concept.
\end{abstract}

\section{INTRODUCTION}

Electroactive structures equipped with embedded actuators and sensors connected to electric circuits, which provide a higher level of multi-functionality such as the capability to harvest energy or to suppress undesired vibrations. In this study we focus on porous materials with periodic structure involving locally controlable $\mathrm{PZ}$ actuators inducing pore deformation. The homogenization based modelling of PZ composites, or foams has been reported in the literature over past two decades, see e.g. $[1,2]$ even in the context of biological tissues or biomaterials $[8,6]$

We consider a fluid-saturated porous medium subjected to deformation waves which generate peristaltic driven flow. The deformation is actuated by piezoelectric elements periodically distributed in the structure and controlled locally by electrodes inducing the electrostatic field. The presented research is aimed to explore functionality of such metamaterial structures by in silico experiments. For this we employ a two-scale modelling 
approach based on the homogenization method [3] combined with the sensitivity analysis. We extend the homogenized model of the fluid-saturated piezo-poroelastic medium equipped with the controlling conductor networks [13] to describe the fluid-structure interaction respecting influence of the deformation of the microconfiguration. The computational model arises from the homogenization of the fluid-saturated porous medium. To treat the large deformation phenomenon, we follow the Eulerian approach leading to the updated-Lagrangian incremental formulation in the two-scale setting $[10,7]$. In the context of locally periodic structures, local cell problems are obtained which provide characteristic responses of the microstructures with respect to macroscopic strains, fluid pressure and electric potentials. Within the homogenization scheme introduced for the incremental fluid-structure interaction problem, the macroscopic nonlinearity of the device is captured using the first order expansions of the homogenized coefficients with respect to macroscopic variables [12], cf. [9]. For this, the sensitivity analysis approach is employed. We present examples of microstructures and results of the simulations as the proof of concept aimed at designing smeared peristaltic pumps in a bulk medium. The computational tools are intended for subsequent two-scale design optimization [4] of local microstructures according to objectives of the macroscopic functionality, namely the fluid pumping.

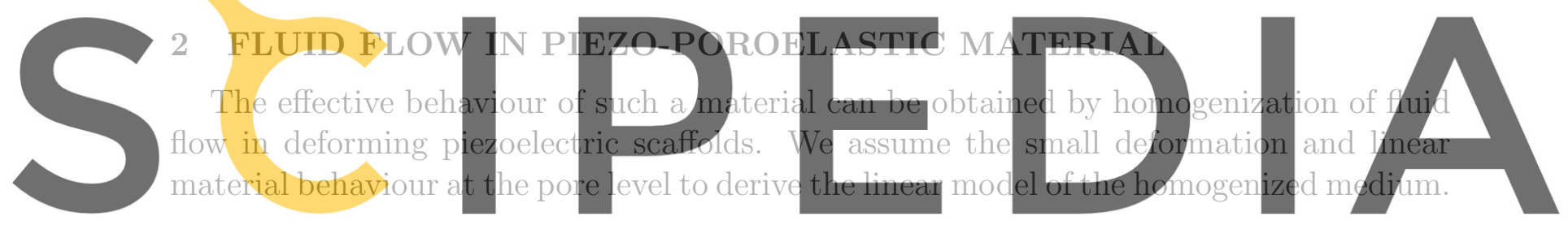

2.1 Micromodel at the pore level

Register for free at https//www.scipedia.com to download the version without the watermark

We consider a quasi-static loading of a piezoelectric skeleton interacting with a viscous

fluid saturating the pores in the skeleton. The two-phase medium is characterized by the pore size $\ell^{\varepsilon} \approx \varepsilon$, where the asymptotic analysis $\varepsilon \rightarrow 0$ leads to a model of the homogenized fluid saturated piezo-poroelastic medium which occupies an open bounded domain $\Omega \subset \mathbb{R}^{3}$. For a given $\varepsilon>0$, the following decomposition of $\Omega$ into the piezoelectric matrix, $\Omega_{m}^{\varepsilon}$, elastic conductive inclusions, $\Omega_{*}^{\varepsilon}$, and fluid-saturated channel parts, $\Omega_{f}^{\varepsilon}$, is considered:

$$
\Omega=\Omega_{f}^{\varepsilon} \cup \Omega_{m}^{\varepsilon} \cup \Omega_{*}^{\varepsilon}, \quad \Omega_{f}^{\varepsilon} \cap \Omega_{m}^{\varepsilon} \cap \Omega_{*}^{\varepsilon}=\emptyset, \quad \text { where } \Omega_{*}^{\varepsilon}=\bigcup_{k} \Omega_{*}^{k, \varepsilon} .
$$

By $\Gamma_{f_{s}}^{\varepsilon}$ we denote the solid-fluid interface. The interface between the piezoelectric matrix and the conductors $\Gamma_{*}^{\varepsilon}$ consists of its subparts $\Gamma_{*}^{k, \varepsilon}=\partial \Omega_{*}^{k, \varepsilon}, k=1, \ldots, k^{*}, k^{*} \geq 2$.

We assume that both the matrix $\Omega_{m}^{\varepsilon}$ and the fluid-filled channels $\Omega_{f}^{\varepsilon}$ are connected domains. The conductive material is distributed as a piecewise connected phase for each index $k$, such that $\Omega_{*}^{k, \varepsilon} \cap \Omega_{*}^{l, \varepsilon}=\emptyset$ when $k \neq l$. The solid occupies domain $\Omega_{m *}=$ $\Omega_{m}^{\varepsilon} \cup \Omega_{*}^{\varepsilon} \cup \Gamma_{*}^{\varepsilon}$ which is constituted by the piezoelectric matrix and by the conductors. 
In the piezoelectric solid, the Cauchy stress tensor $\boldsymbol{\sigma}^{\varepsilon}$ and the electric displacement $\vec{D}^{\varepsilon}=\left(D_{i}^{\varepsilon}\right)$ depend on the strain tensor $\boldsymbol{e}\left(\boldsymbol{u}^{\varepsilon}\right)=\left(\nabla \boldsymbol{u}^{\varepsilon}+\left(\nabla \boldsymbol{u}^{\varepsilon}\right)^{T}\right) / 2$ defined in terms of the displacement field $\boldsymbol{u}^{\varepsilon}=\left(u_{i}^{\varepsilon}\right)$, and on the electric field $\vec{E}^{\varepsilon}=\nabla \varphi^{\varepsilon}$ defined in terms of the electric potential, $E_{i}^{\varepsilon}=\partial_{i}^{x} \varphi^{\varepsilon}$. The following constitutive equations characterize the piezoelectric solid in $\Omega_{m}^{\varepsilon}$,

$$
\begin{aligned}
& \sigma_{i j}^{\varepsilon}\left(\boldsymbol{u}^{\varepsilon}, \varphi^{\varepsilon}\right)=A_{i j k l}^{\varepsilon} \varepsilon_{k l}^{\varepsilon}\left(\boldsymbol{u}^{\varepsilon}\right)-g_{k i j}^{\varepsilon} E_{k}^{\varepsilon}\left(\varphi^{\varepsilon}\right), \\
& D_{k}^{\varepsilon}\left(\boldsymbol{u}^{\varepsilon}, \varphi^{\varepsilon}\right)=g_{k i j}^{\varepsilon} e_{i j}^{\varepsilon}\left(\boldsymbol{u}^{\varepsilon}\right)+d_{k l}^{\varepsilon} E_{l}^{\varepsilon}\left(\varphi^{\varepsilon}\right),
\end{aligned}
$$

where $\mathbb{A}^{\varepsilon}=\left(A_{i j k l}^{\varepsilon}\right)$ is the elasticity fourth-order symmetric positive definite tensor of the solid, where $A_{i j k l}=A_{k l i j}=A_{j i l k}$, the deformation is coupled with the electric field through the 3rd order tensor $\underline{\boldsymbol{g}}^{\varepsilon}=\left(g_{k i j}^{\varepsilon}\right), g_{k i j}^{\varepsilon}=g_{k j i}^{\varepsilon}$ and $\boldsymbol{d}^{\varepsilon}=\left(d_{k l}^{\varepsilon}\right)$ is the permittivity tensor. The conductive solid in $\Omega_{*}^{\frac{\varepsilon}{\varepsilon}}$ is described by its elasticity $\mathbb{A}^{\varepsilon}$ only, since the permittivity infinitely large, so that the electric potential is constant $\varphi^{\varepsilon}=\bar{\varphi}^{k}$ in each of the subparts $\Omega_{*}^{k, \varepsilon}$. In the solid, the straightforward equilibrium conditions including those on the PZ-conductor interface $\Gamma_{*}^{\varepsilon}$ read

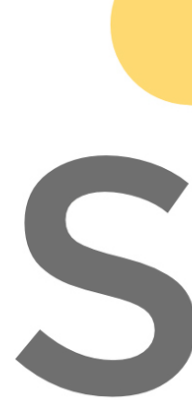

$$
\rho_{s} \ddot{u}^{\varepsilon}-\nabla \cdot \sigma^{\varepsilon}=0, \quad \text { in } \Omega_{* m}^{\varepsilon},
$$$$
\nabla \cdot \vec{D}^{\varepsilon}=0, \quad \text { in } \Omega_{*}^{\varepsilon}
$$

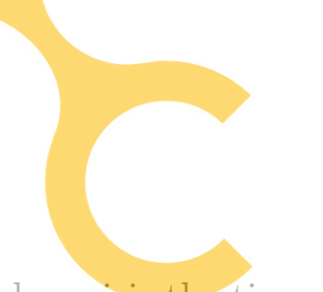

where $a$ is the time derivative
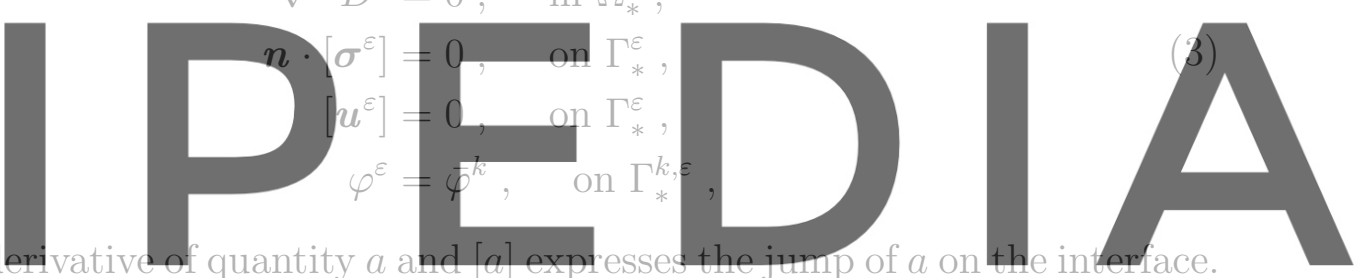

We consider flow of an electrically neutral incompressible Newtonian fluid with viscosity

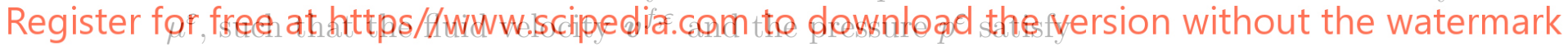

$$
\mu^{\varepsilon} \nabla^{2} \boldsymbol{v}^{f, \varepsilon}-\nabla p^{\varepsilon}-\rho_{f} \frac{\partial}{\partial t} \boldsymbol{v}^{f, \varepsilon}=0, \quad \nabla \cdot \boldsymbol{v}^{f, \varepsilon}, \quad \text { in } \Omega_{f}^{\varepsilon} .
$$

The following conditions describe the interaction of the two phases on $\Gamma_{f s}^{\varepsilon}$,

$$
\boldsymbol{v}^{f, \varepsilon}=\dot{\boldsymbol{u}}^{\varepsilon}, \quad \boldsymbol{n} \cdot \boldsymbol{\sigma}_{f}^{\varepsilon}=\boldsymbol{n} \cdot \boldsymbol{\sigma}_{s}^{\varepsilon}, \text { and } \quad \boldsymbol{n} \cdot \vec{D}^{\varepsilon}=0 \quad \text { on } \Gamma_{f s}^{\varepsilon},
$$

where $\boldsymbol{n}$ is the unit normal w.r.t. surface $\Gamma_{f s}^{\varepsilon}$ and $\boldsymbol{\sigma}_{f}^{\varepsilon}=-p^{\varepsilon} \boldsymbol{I}+\mu^{\varepsilon} \nabla^{2} \boldsymbol{v}^{f, \varepsilon}$ the stress tensors. For further treatment by the asymptotic analysis, it is advantageous to substitute $\boldsymbol{v}^{f, \varepsilon}=\boldsymbol{w}^{\varepsilon}+\dot{\tilde{\boldsymbol{u}}}^{\varepsilon}$, where $\dot{\tilde{\boldsymbol{u}}}^{\varepsilon}$ is a continuous extension of the solid velocity to the fluid domain and $\boldsymbol{w}^{\varepsilon}$ is the relative velocity. The boundary conditions are prescribed on the external boundary $\partial_{\text {ext }} \Omega_{m *}$.

\subsection{Model of the homogenized medium}

The porous structure is formed as a periodic lattice generated by the representative unit cell $Y=]-1 / 2,1 / 2\left[{ }^{3}\right.$, such that $\Omega$ is constituted by copies of real-size cells $Y^{\varepsilon}(\hat{x})=\varepsilon Y+\hat{x}$ 
centered at the lattice nodes $\hat{x} \in \Xi^{\varepsilon}$. The decomposition (1) reflects the one of the representative cell, thus, we have $Y=Y_{m} \cup Y_{*} \cup Y_{f}$, whereby $\Gamma_{f_{s}}=\partial Y_{m *} \cap \partial Y_{f}$ and $\Gamma_{*}=\partial Y_{*}$. We consider formulation (3) with given potentials $\bar{\varphi}^{k}$ for each simply connected domain $\Omega_{*}^{k, \varepsilon}$ occupied by the perfect conductor and represented by $Y_{*}^{k}$ within the cell $Y$, such that $Y_{*}=\bigcup_{k} Y_{*}^{k}, Y_{*}^{k} \cap Y_{*}^{l}=\emptyset$ for $k \neq l$. As the consequence of the strongly controlled electric field due to prescribed potentials $\bar{\varphi}^{k, \varepsilon}=\bar{\varphi}^{k}$ in $\Omega_{*}^{k, \varepsilon}$, a weakly piezoelectric material is required, such that, for given $\overline{\boldsymbol{g}}$ and $\overline{\boldsymbol{d}}$,

$$
\left.\begin{array}{rl}
\boldsymbol{g}^{\varepsilon}(x) & =\varepsilon \overline{\boldsymbol{g}} \\
\overline{\boldsymbol{d}}^{\varepsilon}(x) & =\varepsilon^{2} \overline{\boldsymbol{d}},
\end{array}\right\} \quad \text { in } \Omega_{m}^{\varepsilon}
$$

The elasticity tensor $\mathbb{A}(y)$ is given as a piecewise constant function in the piezoelectric and conductive parts $Y_{m}$ and $Y_{*}$, respectively.

\subsection{Local characteristic responses}

The microproblems of the PZ-poroelastic medium are expressed using the following bilinear forms:
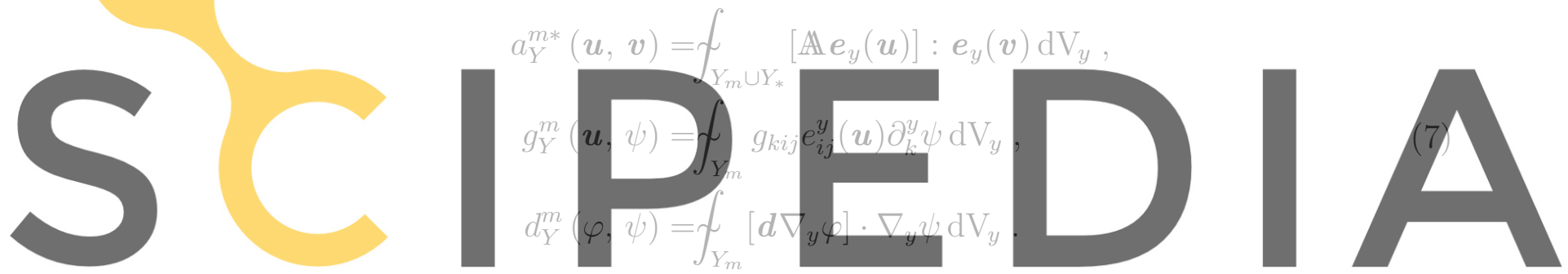

Register for fret at he denote the s.pace of $Y$-periodic functions in the Sobolev space $W_{11.2}^{1.2}(Y)$.

function sets,

$$
\begin{aligned}
H_{\# 0 *}^{1}\left(Y_{m}\right) & =\left\{\psi \in H_{\#}^{1}\left(Y_{m}\right) \mid \psi=0 \text { on } \Gamma_{m *}\right\} \\
U_{\#}^{k}\left(Y_{m}\right) & =\left\{\psi \in H_{\#}^{1}\left(Y_{m}\right) \mid \psi=\delta_{k i} \text { on } \Gamma_{m *}^{i}, i=1,2, \ldots, k^{*}\right\}, \\
\mathbf{H}_{\# 0}^{1}\left(Y_{f}\right) & =\left\{\boldsymbol{\psi} \in \mathbf{H}_{\#}^{1}\left(Y_{m}\right) \mid \boldsymbol{\psi}=0 \text { on } \Gamma_{f_{s}}\right\} .
\end{aligned}
$$

Due to the problem linearity, the scale separation in the limit $\varepsilon \rightarrow 0$ enables to express the local fluctuations of the displacement and electric potential in terms of the characteristic responses which satisfy the following local problems imposed in the solid part $Y_{m *}$.

- Find $\left(\boldsymbol{\omega}^{i j}, \hat{\eta}^{i j}\right) \in \mathbf{H}_{\#}^{1}\left(Y_{m *}\right) \times H_{\# 0 *}^{1}\left(Y_{m}\right)$ for any $i, j=1,2,3$ satisfying

$$
\begin{array}{rlrl}
a_{Y}^{m *}\left(\boldsymbol{\omega}^{i j}+\boldsymbol{\Pi}^{i j}, \boldsymbol{v}\right)-g_{Y}^{m}\left(\boldsymbol{v}, \hat{\eta}^{i j}\right) & =0, & & \forall \boldsymbol{v} \in \mathbf{H}_{\#}^{1}\left(Y_{m *}\right), \\
g_{Y}^{m}\left(\boldsymbol{\omega}^{i j}+\boldsymbol{\Pi}^{i j}, \psi\right)+d_{Y}^{m}\left(\hat{\eta}^{i j}, \psi\right)=0, & \forall \psi \in H_{\# 0 *}^{1}\left(Y_{m}\right),
\end{array}
$$

where $\Pi^{i j}=\left(\Pi_{k}^{i j}\right), i, j, k=1,2,3$ with its components $\Pi_{k}^{i j}=y_{j} \delta_{i k}$. 
- Find $\left(\boldsymbol{\omega}^{P}, \hat{\eta}^{P}\right) \in \mathbf{H}_{\#}^{1}\left(Y_{m *}\right) \times H_{\# 0 *}^{1}\left(Y_{m}\right)$ satisfying

$$
\begin{aligned}
& a_{Y}^{m *}\left(\boldsymbol{\omega}^{P}, \boldsymbol{v}\right)-g_{Y}^{m}\left(\boldsymbol{v}, \hat{\eta}^{P}\right)=-f_{\Gamma_{f s}} \boldsymbol{v} \cdot \boldsymbol{n}^{[f]} \mathrm{d} S_{y}, \quad \forall \boldsymbol{v} \in \mathbf{H}_{\#}^{1}\left(Y_{* m}\right), \\
& g_{Y}^{m}\left(\boldsymbol{\omega}^{P}, \psi\right)+d_{Y}^{m}\left(\hat{\eta}^{P}, \psi\right)=0, \quad \forall \psi \in H_{\# 0 *}^{1}\left(Y_{m}\right),
\end{aligned}
$$

- Find $\left(\hat{\boldsymbol{\omega}}^{k}, \hat{\varphi}^{k}\right) \in \mathbf{H}_{\#}^{1}\left(Y_{m *}\right) \times U_{\#}^{k}\left(Y_{m}\right)$ satisfying, for $k=1,2, \ldots, k^{*}$,

$$
\begin{aligned}
a_{Y}^{m *}\left(\hat{\boldsymbol{\omega}}^{k}, \boldsymbol{v}\right)-g_{Y}^{m}\left(\boldsymbol{v}, \hat{\varphi}^{k}\right)=0, & \forall \boldsymbol{v} \in \mathbf{H}_{\#}^{1}\left(Y_{* m}\right), \\
g_{Y}^{m}\left(\hat{\boldsymbol{\omega}}^{k}, \psi\right)+d_{Y}^{m}\left(\hat{\varphi}^{k}, \psi\right)=0, & \forall \psi \in H_{\# 0 *}^{1}\left(Y_{m}\right) .
\end{aligned}
$$

In the fluid domain $Y_{f}$, the characteristic response $\hat{w}^{k}(\cdot, t) \in \mathbf{H}_{\# 0}^{1}\left(Y_{f}\right)$ and $\hat{\pi}^{k}(\cdot, t) \in$ $H_{\#}^{1}\left(Y_{f}\right)$ satisfies the non-stationary Stokes problem,

\section{$\rho_{f}\left\langle\frac{\partial}{\partial t} \hat{\boldsymbol{w}}^{k}, \boldsymbol{\psi}\right\rangle_{Y_{f}}-\left\langle\hat{\pi}^{k}, \nabla_{y} \cdot \boldsymbol{\psi}\right\rangle_{Y_{f}}+\bar{\mu}\left\langle\nabla_{y} \hat{\boldsymbol{w}}^{k}, \nabla_{y} \boldsymbol{\psi}\right\rangle_{Y_{f}}=\left\langle 1, \psi_{k}\right\rangle_{Y_{f}}, \quad \forall \boldsymbol{\psi} \in \mathbf{H}_{\# 0}^{1}\left(Y_{f}\right)$ \\ $\left\langle q, \nabla_{y} \cdot \hat{w}^{k}\right\rangle_{Y_{f}}=0, \quad \forall q \in L^{2}\left(Y_{f}\right)$}
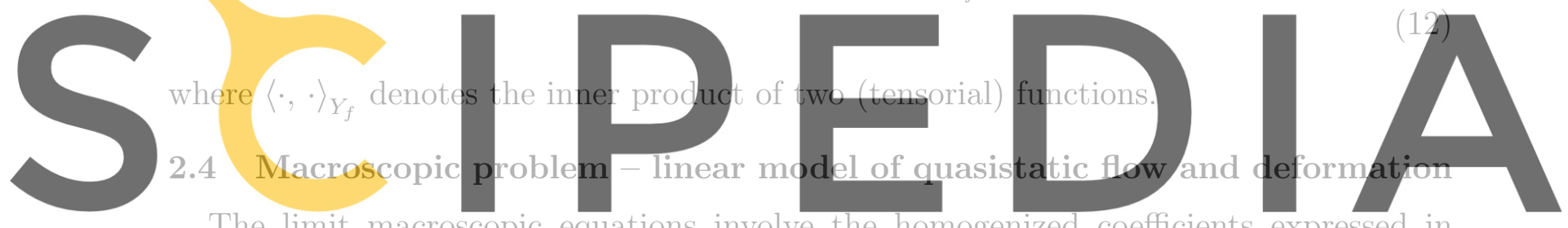

The limit macroscopic equations involve the homogenized coefficients expressed in

Register for free at hetps/ywWW.scipedia.com to download the version without the watermark

$$
\begin{aligned}
A_{k l i j}^{H} & =a_{Y}^{m *}\left(\boldsymbol{\omega}^{i j}+\Pi^{i j}, \omega^{k l}+\Pi^{k l}\right)+d_{Y}^{m}\left(\hat{\eta}^{k l}, \hat{\eta}^{i j}\right), \\
B_{i j}^{H} & =a_{Y}^{m *}\left(\boldsymbol{\omega}^{P}, \boldsymbol{\Pi}^{i j}\right)-g_{Y}^{m}\left(\boldsymbol{\Pi}^{i j}, \hat{\eta}^{P}\right)+\phi \delta_{i j}, \\
M^{H} & =a_{Y}^{m *}\left(\boldsymbol{\omega}^{P}, \boldsymbol{\omega}^{P}\right)+d_{Y}^{m}\left(\hat{\eta}^{P}, \hat{\eta}^{P}\right), \\
H_{i j}^{k} & =a_{Y}^{m *}\left(\hat{\boldsymbol{\omega}}^{k}, \boldsymbol{\Pi}^{i j}\right)-g_{Y}^{m}\left(\boldsymbol{\Pi}^{i j}, \hat{\varphi}^{k}\right), \\
Z^{k} & =-\oint_{\Gamma_{f s}} \hat{\boldsymbol{\omega}}^{k} \cdot \boldsymbol{n}^{[c]} \mathrm{dS}_{y} .
\end{aligned}
$$

Due to the non-stationary Stokes problem (12), the hydraulic permeability depends on time and constitutes the dynamic Darcy law governing the fluid seepage,

$$
K_{i j}^{H}(t)=-\oint_{Y_{f}} \frac{\mathrm{d}}{\mathrm{d} t} \hat{w}_{i}^{j}(t, \cdot) \mathrm{d} \mathrm{V}_{y}
$$

The homogenization procedure leads to a model describing the fluid flow in the deforming PZ-poroelastic medium situated in the macroscopic domain $\Omega \subset \mathbb{R}^{3}$. The model 

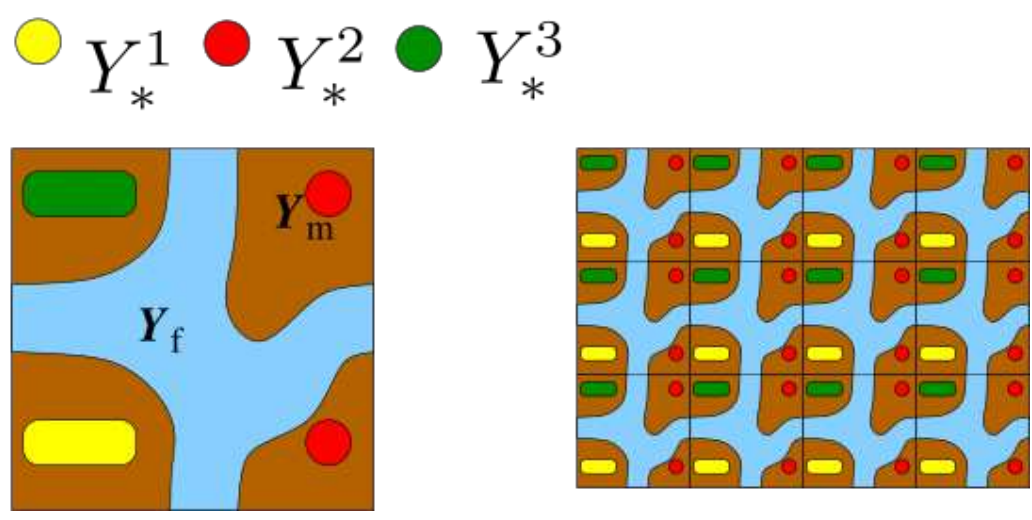

Figure 1: Schematic illustration of the PZ-poroelastic medium with locally controlable electric field.

is represented by following system of equations involving the macroscopic fields, the displacements $\boldsymbol{u}$, the fluid seepage velocity $\boldsymbol{w}$, and the pore fluid pressure $p$ satisfying
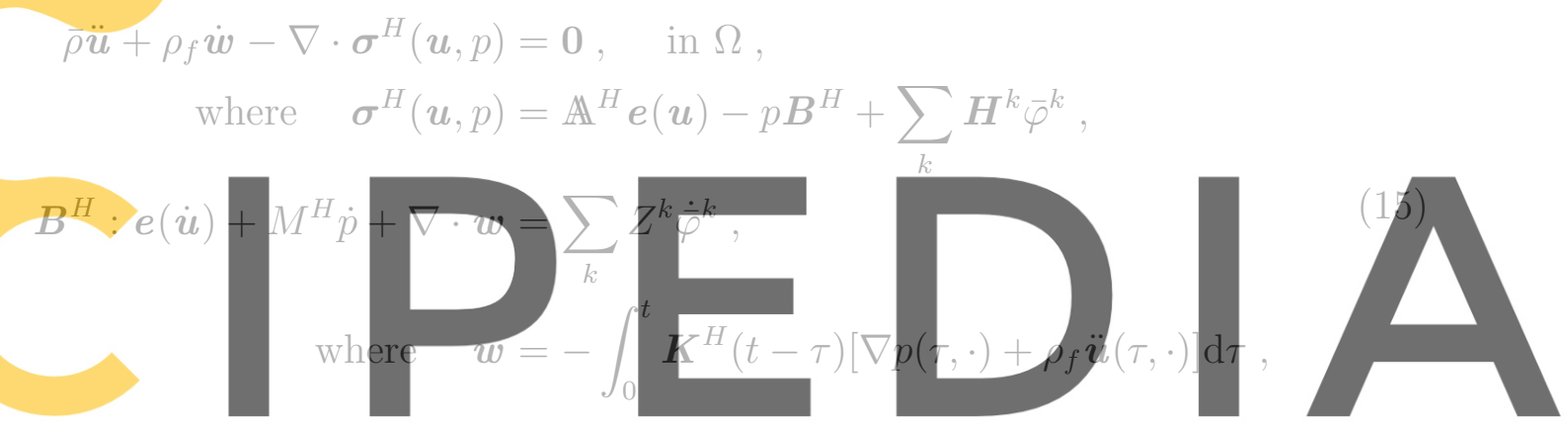

whereby the voltage potentials $\bar{\varphi}^{k}(t, x), k=1, \ldots, k^{*}$ are assumed to be known functions

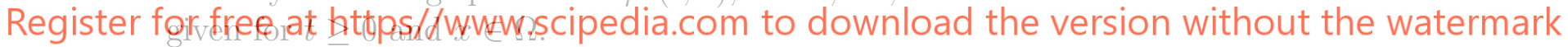

Boundary $\partial \Omega$ is decomposed into disjoint parts $\Gamma_{i}, i=0,1,2$, such that $\partial \Omega=\Gamma_{0} U$

$\Gamma_{1} \cup \Gamma_{2}$. The following boundary conditions can be considered on $\partial \Omega$

$$
\begin{array}{r}
p=\bar{P}_{i} \text { on } \Gamma_{i}, i=1,2, \\
\boldsymbol{n} \cdot \boldsymbol{w}=0 \text { on } \Gamma_{0}, \\
\boldsymbol{u}=\mathbf{0} \text { on } \Gamma_{1}, \\
\boldsymbol{n} \cdot \boldsymbol{\sigma}=\mathbf{0} \text { on } \Gamma_{0}, \\
\boldsymbol{n} \cdot \boldsymbol{\sigma}=-\bar{P}_{2} \boldsymbol{n} \text { on } \Gamma_{2} .
\end{array}
$$

Problem formulation Let both the fields $(\boldsymbol{u}, p)$ vanish at $t=0$ in $\Omega$. For given $P^{k}(t)$ functions of $t>0$, whereby $P^{k}(0)=0$ for $k=1,2$ and given electric actuation by voltages $\left\{\bar{\varphi}^{k}(t, x)\right\}_{k}$ defined for $(t, x) \in[0, T] \times \Omega$, with $T>0$, whereby $\bar{\varphi}^{k}(0, \cdot)=0$ in $\Omega$, find a solution $(\boldsymbol{u}, p)(t, x)$ satisfying (15)-(16). 
To achieve the merits of the peristaltic pumping, we are interested in the fluid transport of the controlable porous medium. For the BCs (16), the global flow rate at time $t$ is

$$
\dot{Q}(t)=\int_{\Gamma_{2}} \boldsymbol{w}(t, \cdot) \cdot \boldsymbol{n} \mathrm{dS}_{y} .
$$

\section{GEOMETRICAL NONLINEARITY APPROXIMATION}

Since the peristaltic flow is driven by the pore deformation, it is crucial to capture the influence of the deformation on the permeability and other effective model parameters, though it is derived using the linear kinematics framework. As a compromise between the linear modelling leading to model (15) and a fully nonlinear treatment, cf. [7], we suggest to apply the approach proposed in $[12]$ which is based on the shape sensitivity analysis, cf. [14].

\subsection{Deforming microstructure}

Due to the characteristic responses computed in (10)-(11), deformed geometry is represented locally by $\tilde{Y}(x)=Y+\left\{\boldsymbol{u}^{\text {mic }}(x, Y)\right\}$ for any $x \in \Omega$, which enables to introduce the perturbed microconfiguration $\tilde{\mathcal{M}}\left(u^{\text {mic }}(x, \cdot), Y\right)$. The micro-displacement field which
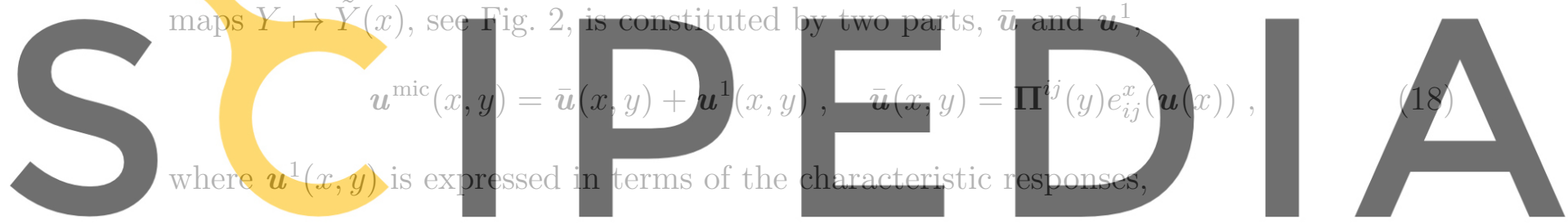

Register for free at https//www.scipedia.com to download the version without the watermark

Recall that $\omega, \hat{\eta}$ and $\hat{\varphi}$ are $Y$-periodic, representing the displacements in the entire solid part, $Y_{m *}=Y_{m} \cup Y_{*}$.

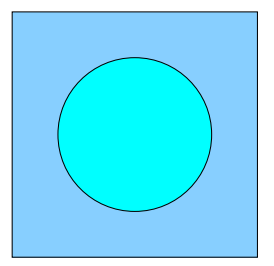

$Y$

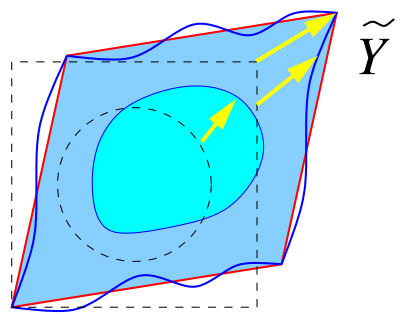

Figure 2: Illustration of the cell deformation due to the displacements $\boldsymbol{u}^{\text {mic }}$. 


\subsection{Deformation dependent effective parameters}

Using the micro-displacement defined in (18) and (19), the homogenized coefficients can be computed using the perturbed microconfigurations represented by the deformed geometry of $\tilde{Y}(x)$, depending on the macroscopic coordinate. Although the microstructure is perturbed from its periodic structure, the homogenization procedure can still be applied and the homogenized coefficients denoted by $\mathbb{H}(\tilde{\mathcal{M}}(\tilde{\boldsymbol{u}}, Y))$, in a generic sense, can be computed for the perturbed geometry determined by the two-scale field $\boldsymbol{u}^{\text {mic }}(x, y)$, $y \in \tilde{Y}(x)$. To avoid solving the local problems (10)-(11) redefined in $\tilde{Y}(x)$, we pursue the treatment reported in [12]. By virtue of the split (19), the perturbed coefficients $\mathbb{H}(\tilde{\mathcal{M}}(\tilde{\boldsymbol{u}}, Y)) \approx \tilde{\mathbb{H}}\left(\boldsymbol{e}(\boldsymbol{u}), p,\left\{\bar{\varphi}^{k}\right\}\right)$ can be approximated using the first order expansion formulae which have the generic form applicable to each of the homogenized coefficients,

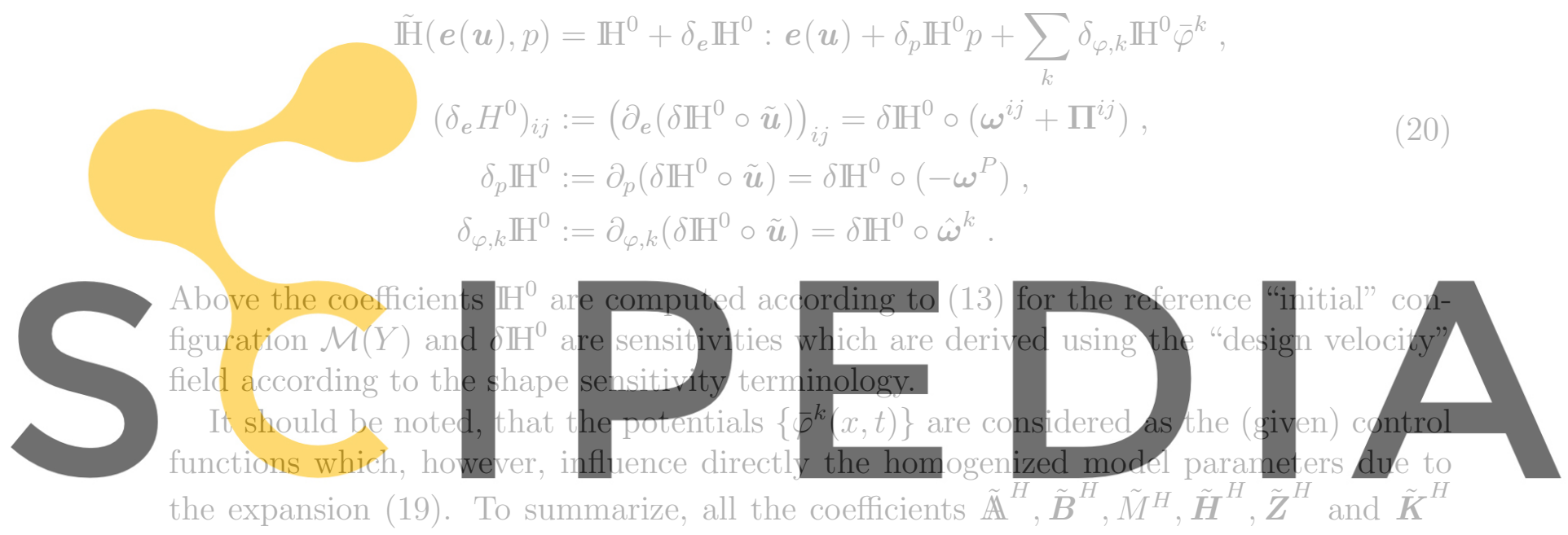

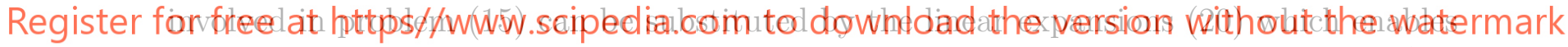
to respect the influence of the microstructure geometry associated with the spatial (deformed) configuration. Althouth the two-scale problem becomes nonlinear, for a periodic initial configuration all the characeristic responses and the sensitivities are computed for the unperturbed cell $Y$, thus, independently of the macroscopic solutions.

\subsection{NUMERICAL ILLUSTRATION OF THE PERISTALTIC FLOW}

For illustration of the peristaltic flow generated by the voltage-controlled PZ-poroelastic material we consider a 3D slab, i.e. an elongated hexahedron, see Fig. 4, its largest dimension (aligned with axis $x_{1}$ ) is $0.1 \mathrm{~m}$. A composite made of an elastic material and a PZT-5 piezoelectric material, cf. [5], interacts with water in the channels; the microstructure with two electrodes is depicted in Fig. 3. The boundary conditions are prescribed according to (16). The electric potential $\bar{\varphi}^{2}=0$, while $\bar{\varphi}^{1}$ is given as

$$
\bar{\varphi}^{1}(t, x)=\bar{\Phi} \cos (\alpha) \operatorname{sgn}\{\cos (\alpha)\}, \quad \text { with } \quad \alpha=\pi\left(x_{1}+\xi(t)\right) / b \text { and } \xi(t)=c t+b / 2
$$

where $c, b, \bar{\Phi}$ are given constants. 

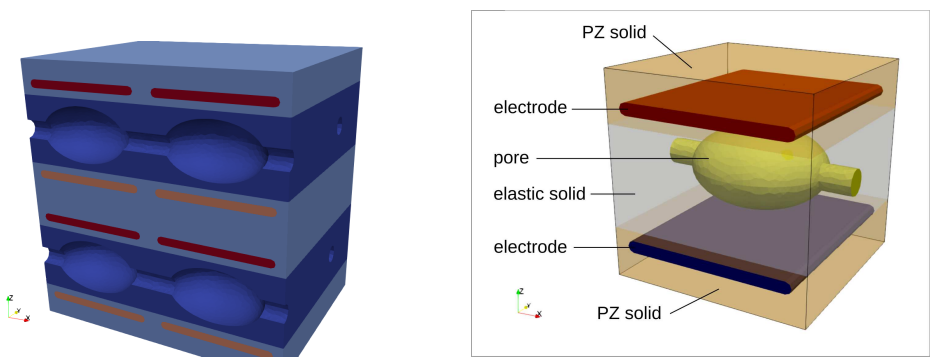

Figure 3: The periodic microsctructure with two electrodes (left). The representative unit cell $Y$ (right).

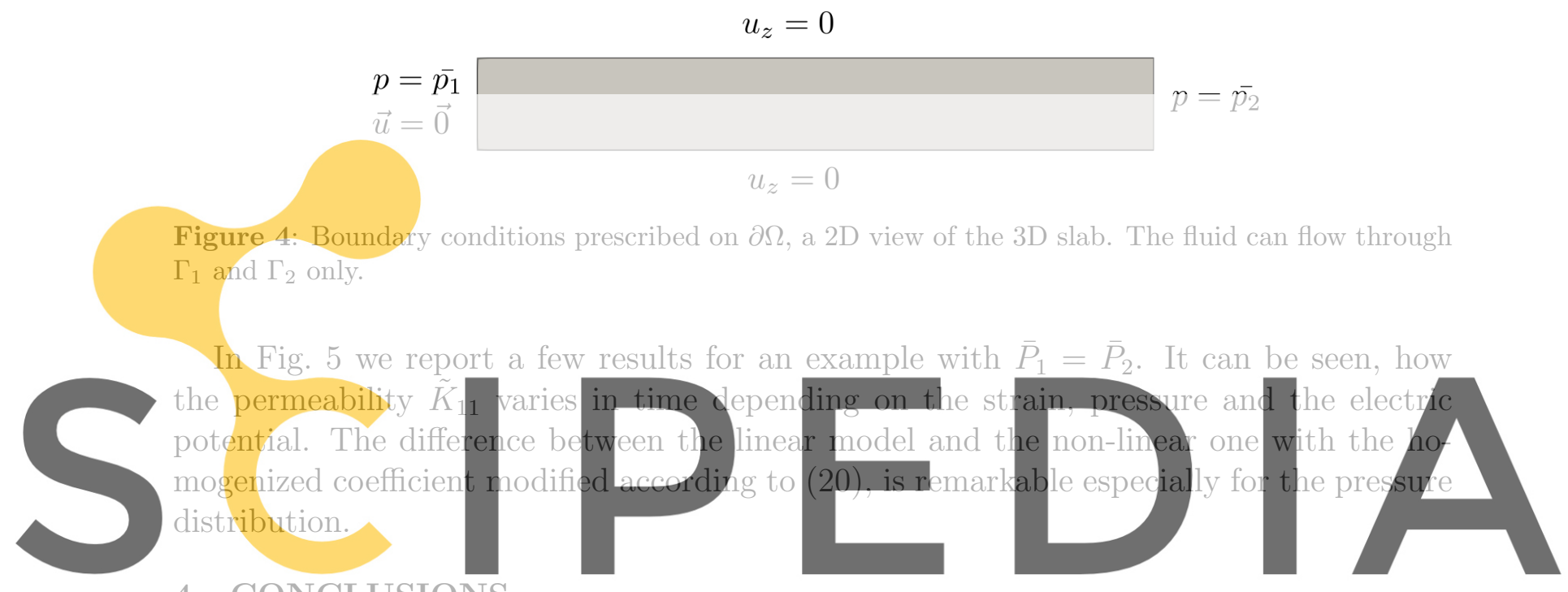

4 CONCLUSIONS

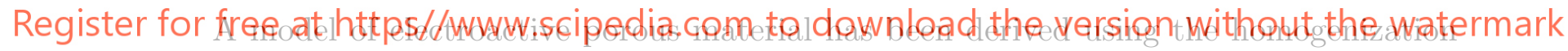

of the linearized fluid-structure interaction problem. The piezoelectric components of the

skeleton are actuated due to electrodes embedded in the microstructure which enable to induce electric field fluctuating at the microstructure level. However, in this study, the peristaltic flow in is achieved due to the piezoelectric effect in response to the propagating macroscopic electric potential wave. To respect deformation-dependent effective properties of the homogenized material, the sensitivity analysis approach has been applied. It leads to a computationally efficient numerical scheme for solving the nonlinear problem. Numerical results show a significant influence of this nonlinearity when compared to the fully linear model with fixed homogenized coefficients.

In the further research, we intend to focus on the optimization of the peristaltic flow [4] by means of the microstructure design and the control. Also the self-contact at the pore level which would enable to minimize the fluid reflux and thus to enhance the pumping efficiency presents another challenging issue [11]. 

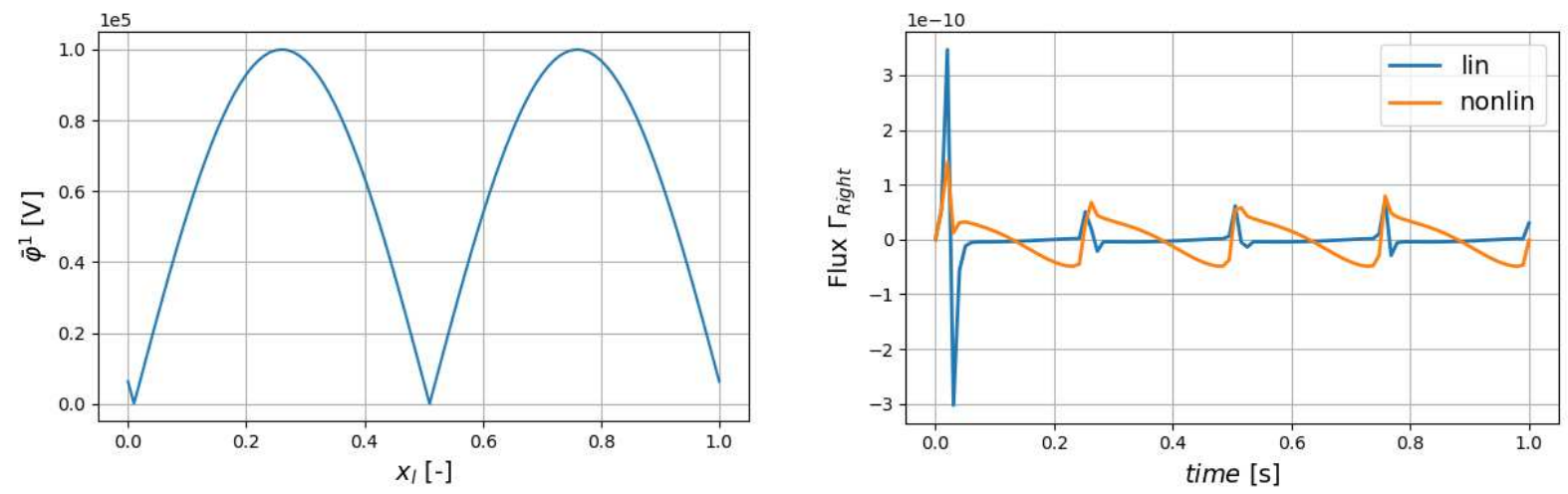

voltage distribution $\bar{\varphi}^{-1}(\hat{t}, \cdot)$ at

flux rate at $\Gamma_{1}$ in time

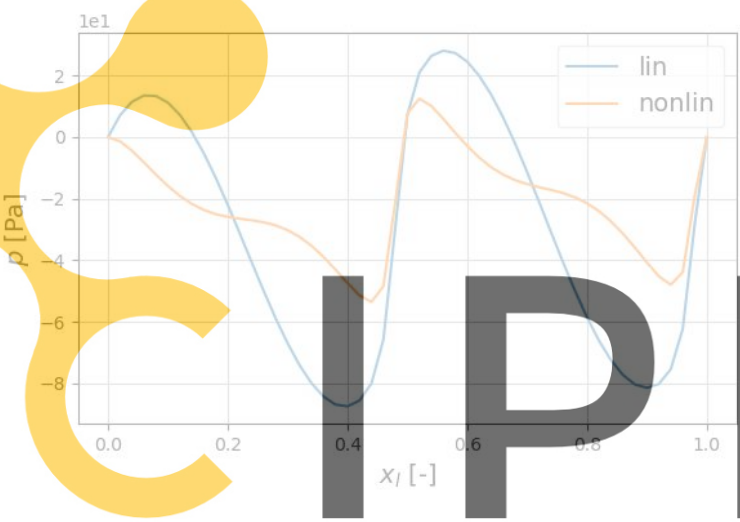

pressure $p(\hat{t}, \cdot)$

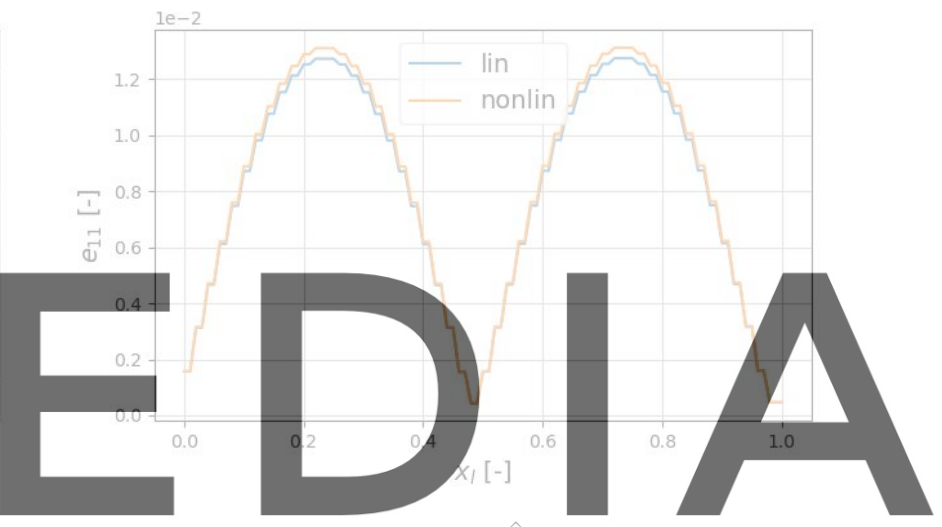

strain $e_{11}(\hat{t}, \cdot)$

Register for free at https//www.scipedia.com to download the version without the watermark

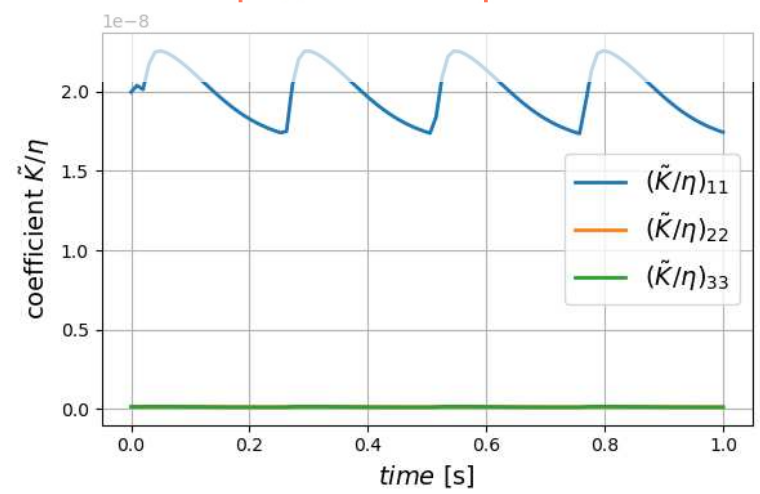

Permeability $\tilde{K}_{i j}$, variation in time.

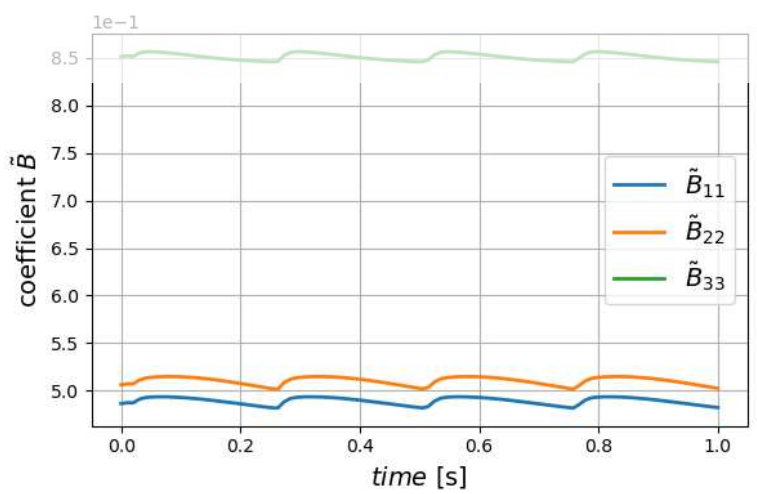

Biot coefficients $\tilde{B}_{i j}$, variation in time.

Figure 5: Results computed by the homogenized model; comparison between the linear model and its nonlinear modification. $\hat{t}=0.5 \mathrm{~s}, x_{1} \in[0,1]$. The time records at $x_{1}=0.1$. 


\section{Acknowledgement}

The research has been supported by the grant projects GACR 19-04956S and GACR 21-16406S of the Czech Science Foundation, and in a part by the European Regional Development Fund-Project "Application of Modern Technologies in Medicine and Industry" (No. CZ.02.1.01/0.0/ 0.0/17 048/0007280) of the Czech Ministry of Education, Youth and Sports.

\section{REFERENCES}

[1] G. M. Ayuso, M. I. Friswell, S. Adhikari, H. H. Khodaparast, and H. Berger. Homogenization of porous piezoelectric materials. International Journal of Solids and Structure, 113-114:218-229, 2017.

[2] R.O. Caballero-Pérez, J. Bravo-Castillero, and L.D. et al. Pérez-Fernández. Computation of effective thermo-piezoelectric properties of porous ceramics via asymptotic homogenization and finite element methods for energy-harvesting applications. Arch Appl Mech, 90:1415-1429, 2020.

[3] D. Cioranescu, A. Damlamian, and G. Griso. The periodic unfolding method in homogenization. SIAM Journal on Mathematical Analysis, 40(4):1585-1620, 2008.

[4] D. Hübner, E. Rohan, V. Lukeš, and M. Stingl. Optimization of the porous material described by the Biot model. Int. J. Solids Struct., 156-157:216-233, 2019.

[5] Y. Koutsawa, S. Belouettar, A. Makradi, and H. Nasser. Sensitivities of effective properties computed using micromechanics differential schemes and high-order taylor series: Application to piezo-polymer composites. Mech. Res. Commun., 37(5):489494, 2010.

[6] T. Lemaire, E. Capiez-Lernout, J. Kaiser, S. Naili, E. Rohan, and V. Sansalone. A multiscale theoretical investigation of electric measurements in living bone. piezoelectricity and electrokinetics. Bulletin of Mathematical Biology, 73(11):2649-2677, 2011.

[7] V. Lukeš and E. Rohan. Homogenization of large deforming fluid-saturated porous structures, 2020. http://arxiv.org/abs/2012.03730.

[8] B. Miara, E. Rohan, M. Zidi, and B. Labat. Piezomaterials for bone regeneration design - homogenization approach. J. Mech. Phys. Solids, 53:2005, 2529-2556.

[9] E. Rohan. Sensitivity strategies in modelling heterogeneous media undergoing finite deformation. Math. Comput. Simulat., 61(3-6):261-270, 2003.

[10] E. Rohan. Modelling large deformation induced microflow in soft biological tissues. Theor. and Comp. Fluid Dynamics, 20:251-276, 2006. 
[11] E. Rohan and J. Heczko. Homogenization and numerical modelling of poroelastic materials with self-contact in the microstructure. Computers 85 Structures, 230:106086, 2020 .

[12] E. Rohan and V. Lukeš. Modeling nonlinear phenomena in deforming fluid-saturated porous media using homogenization and sensitivity analysis concepts. Applied Mathematics and Computation, 267:583-595, 2015.

[13] E. Rohan and V. Lukeš. Homogenization of the fluid-saturated piezoelectric porous media. International Journal of Solids and Structures, 147:110-125, 2018.

[14] E. Rohan and B. Miara. Homogenization and shape sensitivity of microstructures for design of piezoelectric bio-materials. Mechanics of Advanced Materials and Structures, 13(6):473-485, 2006. 